\title{
Multimodal prehabilitation in colorectal cancer patients to improve functional capacity and reduce postoperative complications: the first international randomized controlled trial for multimodal prehabilitation
}

Stefanus van Rooijen ${ }^{1}$ (D) Francesco Carli², Susanne Dalton³ ${ }^{3}$ Gwendolyn Thomas ${ }^{1}$, Rasmus Bojesen ${ }^{4}$, Morgan Le Guen ${ }^{5}$, Nicolas Barizien ${ }^{6}$, Rashami Awasthi ${ }^{2}$, Enrico Minnella ${ }^{2}$, Sandra Beijer ${ }^{7}$, Graciela Martínez-Pallii, Rianne van Lieshout ${ }^{9}$, Ismayil Gögenur ${ }^{4}$, Carlo Feo ${ }^{10}$, Christoffer Johansen ${ }^{3,11}$, Celena Scheede-Bergdahl ${ }^{4,12}$, Rudi Roumen', Goof Schep ${ }^{13}$ and Gerrit Slooter ${ }^{1,14^{*}}$

\begin{abstract}
Background: Colorectal cancer (CRC) is the second most prevalent type of cancer in the world. Surgery is the only curative option. However, postoperative complications occur in up to $50 \%$ of patients and are associated with higher morbidity and mortality rates, lower health related quality of life (HRQoL) and increased expenditure in health care. The number and severity of complications are closely related to preoperative functional capacity, nutritional state, psychological state, and smoking behavior. Traditional approaches have targeted the postoperative period for rehabilitation and lifestyle changes. However, recent evidence shows that the preoperative period might be the optimal moment for intervention. This study will determine the impact of multimodal prehabilitation on patients' functional capacity and postoperative complications.

Methods/design: This international multicenter, prospective, randomized controlled trial will include 714 patients undergoing colorectal surgery for cancer. Patients will be allocated to the intervention group, which will receive 4 weeks of prehabilitation (group 1, prehab), or the control group, which will receive no prehabilitation (group 2, no prehab). Both groups will receive perioperative care in accordance with the enhanced recovery after surgery (ERAS) guidelines. The primary outcomes for measurement will be functional capacity (as assessed using the six-minute walk test (6MWT)) and postoperative status determined with the Comprehensive Complication Index (CCI). Secondary outcomes will include HRQoL, length of hospital stay (LOS) and a cost-effectiveness analysis.

(Continued on next page)
\end{abstract}

\footnotetext{
* Correspondence: g.slooter@mmc.nl

'Department of Surgical Oncology, Máxima Medical Center, Veldhoven, the Netherlands

${ }^{14}$ Department of Surgery, Máxima Medical Center, P.O. Box 7777, Veldhoven, the Netherlands

Full list of author information is available at the end of the article
}

(c) The Author(s). 2019 Open Access This article is distributed under the terms of the Creative Commons Attribution 4.0 International License (http://creativecommons.org/licenses/by/4.0/), which permits unrestricted use, distribution, and reproduction in any medium, provided you give appropriate credit to the original author(s) and the source, provide a link to the Creative Commons license, and indicate if changes were made. The Creative Commons Public Domain Dedication waiver (http://creativecommons.org/publicdomain/zero/1.0/) applies to the data made available in this article, unless otherwise stated. 
(Continued from previous page)

Discussion: Multimodal prehabilitation is expected to enhance patients' functional capacity and to reduce postoperative complications. It may therefore result in increased survival and improved HRQoL. This is the first international multicenter study investigating multimodal prehabilitation for patients undergoing colorectal surgery for cancer.

Trial registration: Trial Registry: NTR5947 - date of registration: 1 August 2016.

Keywords: Prehabilitation, Colorectal surgery, Functional capacity, Enhanced recovery after surgery, Comprehensive complication index, Postoperative complications, Colorectal cancer

\section{Background}

Colorectal cancer (CRC) is the second most prevalent type of cancer in the world, with over 1.4 million cases and 693.900 deaths a year [1]. The only way to cure this condition is by surgical removal of the tumor. However, postoperative complications occur in up to $50 \%$ of patients and they are associated with higher morbidity and mortality rates, increased expenditure on health care and poorer health related quality of life (HRQoL) [2-4]. Major surgery brings a 20 to $40 \%$ reduction in physiological and functional capacity, even in absence of complications [5]. This diminished reserve increases the level of fatigue up to months after hospital discharge [6]. Only $40 \%$ of patients return to their preoperative baseline functional capacity (as measured by VO2 peak). Moreover, a proportion of colorectal cancer patients are eligible for (neo)adjuvant which is known to further compromise functional recovery and survival $[7,8]$.

There is emerging evidence suggesting that many of the negative effects of major surgery can be reduced through the attenuation of surgical stress [9]. Efforts to improve the recovery process have primarily focused on the intraoperative factors (such as minimally invasive surgery and afferent neural blockade [10]) and postoperative interventions (examples being "fast track" early nutrition and mobilization [11]). The latter protocols have been designed to facilitate the return of functional activities and accelerate convalescence. However, the postoperative period may not be the best time to ask surgical patients to make significant changes in their nutrition and exercise since patients are tired and concerned about perturbing the healing process. As well as anxious about possible additional treatments for their underlying condition. The preoperative period may in fact be a better time to intervene in the factors that contribute to recovery, both physical and mental, and alleviate some of the emotional distress associated with the anticipation of surgery and the recovery process [12-14].

The process of improving the functional capacity of the individual in order to enable them to withstand an incoming stressor has been termed prehabilitation $[15,16]$. Although some approaches have focused on education to prepare patients for procedures [17], few steps have been taken to systematically enhance functional capacity before surgery. With most studies performing single modal interventions only. To investigate the impact of preoperative exercise on the recovery of functional capacity after colorectal surgery, a few pilot studies have been performed which show promising results [14-16, 18, 19].

Subgroup analysis of the study of Carli et al., showed that patients whose functional exercise capacity improved preoperatively, recovered relatively well in the postoperative period - regardless of the exercise technique [20]. However, one-third of patients deteriorated preoperatively despite the exercise regimen, and these patients were also at greater risk of prolonged recovery after surgery. Poor preoperative physical function (fatigue, malnutrition and physical performance) and the presence of anxiety and depression were also significant confounding predictors of prolonged recovery [21-25]. These results suggest that exercise training alone is not sufficient to attenuate the stress response in all patients and that it is also important to address factors such as nutrition and coping behavior that promote beneficial adaptation to training. Gillis et al. conducted a pilot study that showed that significant changes in postoperative functional exercise capacity can be achieved with a prehabilitation program [26]. However, they did not address the clinically relevant relationship between preoperative functional capacity (an increase of more than $20 \mathrm{~m}$ on the six-minute walk test $(6 \mathrm{MWT}))$, and the postoperative outcome (comprehensive complication index (CCI) reduction of $30 \%)$. If functional capacity can be improved preoperatively, we may expect a reduction in postoperative complications.

Since it has been established that the number and severity of complications are closely related to preoperative functional capacity, nutritional status, smoking behavior and psychological well-being, there has been increasing interest in targeting these issues with a multimodal intervention program [15]. From a physiological point of view and based on limited practical experience, it seems feasible to achieve clinically relevant effects during the period of 4-5 weeks between diagnosis and 
operation [14, 15, 27]. However, this can only be achieved with targeted interventions that include exercise, nutrition, stopping smoking, and psychological support.

\section{Study objectives}

The general aim of this study is to investigate whether multimodal prehabilitation could enhance postoperative outcome using the CCI and 6MWT. Secondary outcomes will include patient reported outcome measures (PROMs) such as HRQoL and depression and anxiety scores, functional capacity measurements, nutritional and smoking status, length of hospital stay, study compliance, patients' satisfaction and a cost-effectiveness analysis.

\section{Methods}

This is an international multicenter, randomized controlled trial with two study groups. Written informed consent will be obtained from all patients. The trial will be conducted according to the rules of Good Clinical Practice and a Data Safety Monitoring Board (DSMB) has been appointed to monitor (serious) adverse events. The Netherlands Comprehensive Cancer Organisation will be responsible for quality control and data management. Ethical approval for this study was granted by the Medical Ethics Committee of the Máxima Medical Center (Veldhoven, the Netherlands) under reference number W16.100/NL58281.015.16. Important protocol modifications will be addressed to relevant parties.

\section{Study population}

Adult patients ( $>18$ years) undergoing elective colorectal resection for cancer are eligible for inclusion. We will include 714 patients: 357 in each arm. We expect a dropout rate of $10 \%$ based on previous pilot studies. The estimated duration of the recruitment period is two years. Exclusion criteria are metastatic disease known preoperatively, paralysis or patients with mobility problems (who are unable to exercise), premorbid conditions or orthopedic impairments that contraindicated exercise, cognitive disabilities, chronic renal failure (dialysis or creatinine $>250 \mathrm{mmol}$ ), ASA score 4 or higher, and illiteracy (inability to read and understand the language of the country where the study will be performed).

\section{Participating centers}

Patients from the Máxima Medical Center (coordinating hospital, Eindhoven-Veldhoven, the Netherlands), the Montréal General Hospital (Montréal, McGill, Canada), Zealand University Hospital (Zealand Region, Denmark), Foch Hôpital (Paris, France), the Saint Anna University Hospital of Ferrara (Ferrara, Italy), and Hospital Clinic de Barcelona (Barcelona, Spain), will be included in this study.

\section{Randomization}

Patients will be block randomized with a 1:1 allocation by means of randomization software (Research Manager clinical trial data management system, Deventer, the Netherlands), stratified by study sites, tumor location and neoadjuvant treatment. Patients will be allocated either to the intervention group, which will receive 4 weeks of prehabilitation, or to the control group, which will receive no prehabilitation.

In all participating centers, both the investigator and the surgeon responsible will verify eligibility. If the indication for surgery is established, patients will be screened by the medical research team for health conditions that prohibit participation in the program. They will then be called by the research investigator and an appointment will be made to provide written and oral information about the trial during a scheduled outpatient appointment. Patients will be given enough time to enquire about the details of the trial and to decide whether or not they wish to participate. Patients will be required to sign the informed consent form in the presence of the surgeon or investigator. A participant flow diagram is shown in Fig. 1. After the study has been explained and consent is obtained, there will be a multidisciplinary assessment. Based on intake by the sports physician, the physiotherapist/kinesiologist, the nutritionist and the case manager/psychologist, an individual prehabilitation program will be started during four weeks in the intervention group.

\section{Study outline}

Routine standard preoperative and postoperative clinical care in the participating institutions do not currently include special nutrition, exercise and psychological support to cope with anxiety before surgery. As usual, patients are evaluated in the preoperative clinic before surgery to determine whether they are fit for surgery and to adjust their medication for co-morbid conditions. It is not common practice after surgery to offer them an in-hospital exercise program but patients are given generic instructions by the surgeons about mobilization and returning to normal activities. Some patients may be referred to a physiotherapist by their surgeon. Nutrition, exercise and coping strategies will be introduced solely for research purposes. All patients will be screened four weeks before surgery to capture insufficient hemoglobin levels (thresholds in Canada: $>11.2 \mathrm{~g} / \mathrm{dl}$, Europe: $>7 \mathrm{mmol} / \mathrm{l}$ ). Hemoglobin levels in patients with iron insufficiency will be optimized using iron injections (ferinject).

Perioperative care will be based on a standardized, multi-element, evidence-based, comprehensive, ERAS guideline in line with the consensus review of optimal care for patients undergoing colorectal surgery [27]. The guideline will be applied in all participating centers to improve generalizability. 


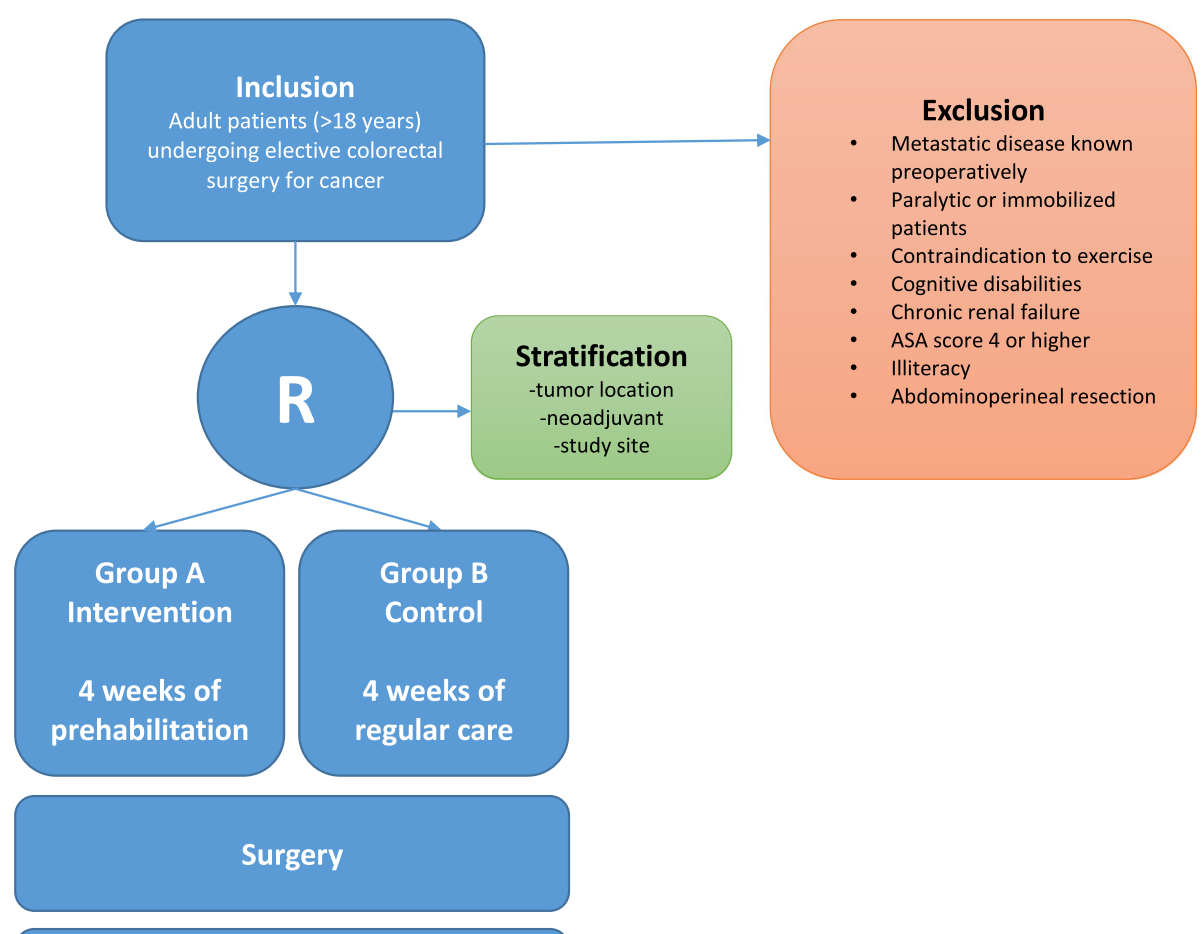

Follow up at 4, 8 weeks and 1 year

Fig. 1 Flow diagram for study participants

The diagnostic work-up for patients with a tumor suspected for malignancy at colonoscopy will be finalized within one week while awaiting definitive pathology. An individual treatment strategy will then be proposed by the multidisciplinary team. Patients that meet the criteria for the trial will be scheduled approximately five weeks after the final diagnosis. This schedule allows for the implementation of a four-week prehabilitation program.

The multidisciplinary multimodal prehabilitation program is composed of 4 elements: exercise training, nutritional intervention, smoking cessation and psychological support. The exact interventions are shown in Table 1 and described in detail below:

\section{Exercise program}

An exercise specialist (kinesiologist, sport physician) will assess the patients' mobility and his/her capacity to exercise using a cardiopulmonary exercise test (CPET). The CPET values will be used to establish an individualized dosing of training embedded in a standardized training program [28].

- The interval training duration is $28-32 \mathrm{~min}$ and performed with 4 min of warm-up at moderate intensity, 4 intervals of High intensity ( $2-3 \mathrm{~min})$ and 4 intervals of Moderate intensity $(4 \mathrm{~min})$.
- The workload is dosed at the wattage corresponding to $90 \%$ the peak wattage as attained by the CPET. This is aimed to result in a metabolic response ranging from 85 to $100 \%$ of VO2peak during the high intensity intervals. This corresponds with a heart-rate of $85-100 \%$ of maximal heart rate, and a Borg score of $15-17$. Moderate intensity is set at $30 \%$ of peak wattage of the CPET test. This is aimed to have recovery at a level around or just below Aerobic Threshold.

- If the patient is unable to complete the high-intensity bout for 4 periods of $2 \mathrm{~min}$ (=less than 8 min' exercise in the high intensity range), the intensity is reduced by $10 \%$. The intensity is reduced further - in steps of $10 \%$ - until the patient can complete the 4 bouts of at least $2 \mathrm{~min}$. If the patient is able to complete all 4 high intensity bouts for $3 \mathrm{~min}$ (12 $\mathrm{min}$ in the high intensity range) the load is increased with $10 \%$. If necessary fine-tuning can be done using steps of $5 \%$.

In addition to the preoperative exercise program, patients will receive information about breathing techniques to prevent pneumonia.

Patients in the intervention group will have three supervised in-hospital training sessions per week during four weeks. This includes interval training and resistance training. 


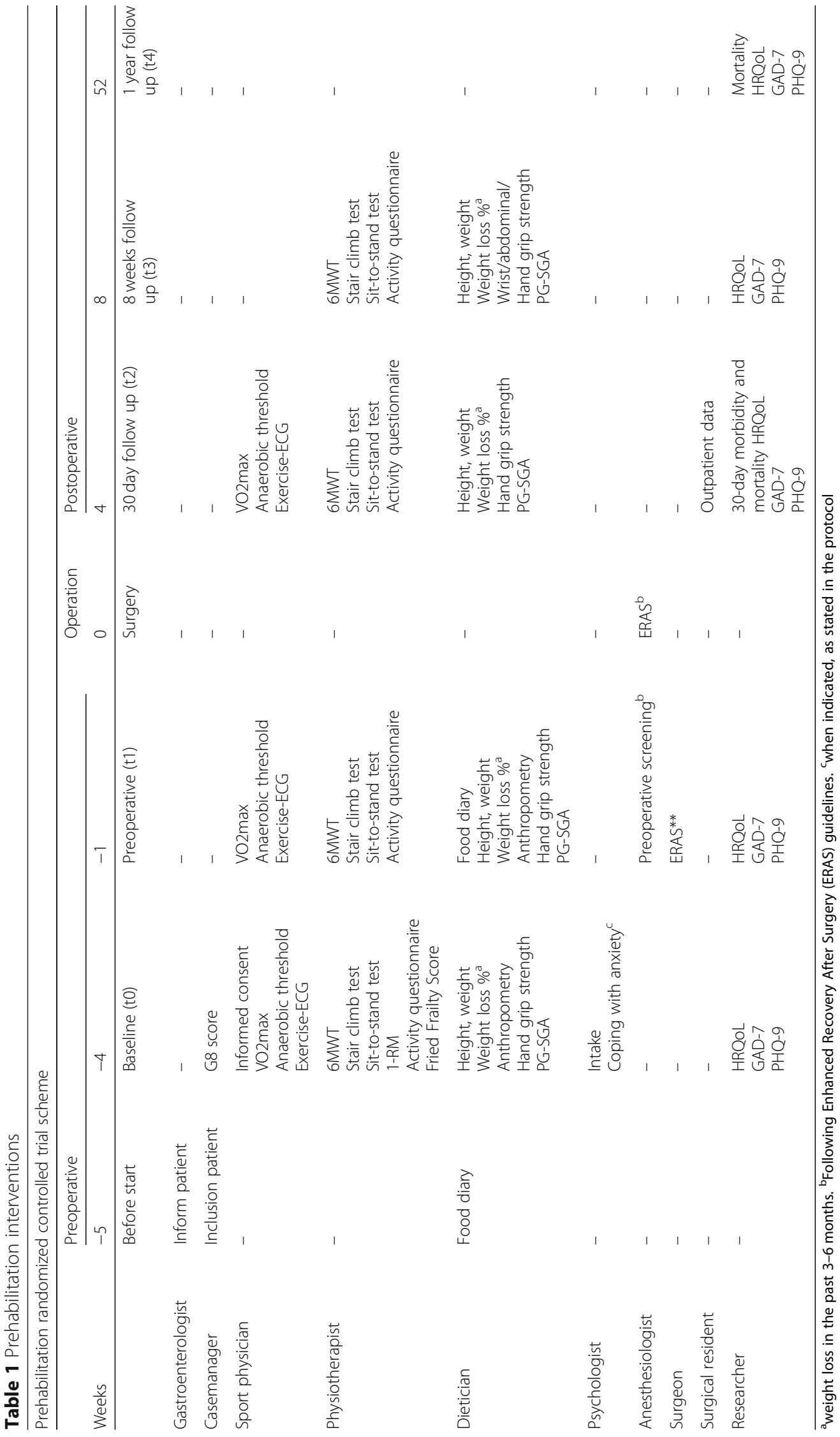


The interval training duration is $28-32 \mathrm{~min}$ and performed with $4 \mathrm{~min}$ of warm-up at moderate intensity, 4 intervals of High intensity (2-3 min) and 4 intervals of Moderate intensity (4 min).

The workload is dosed at the wattage corresponding to $90 \%$ the peak wattage as attained by the CPET. This is aimed to result in a metabolic response ranging from 85 to $100 \%$ of VO2peak during the high intensity intervals. This corresponds with a heart-rate of $85-100 \%$ of maximal heart rate, and a Borg score of 15-17 Moderate intensity is set at $30 \%$ of peak wattage of the CPET test. This is aimed to have recovery at a level around or just below Aerobic Threshold.

If the patient is unable to complete the high-intensity bout for 4 periods of $2 \mathrm{~min}$ (=less than 8 min' exercise in the high intensity range), the intensity is reduced by $10 \%$. The intensity is reduced further - in steps of $10 \%$ - until the patient can complete the 4 bouts of at least $2 \mathrm{~min}$. If the patient is able to complete all 4 high intensity bouts for $3 \mathrm{~min}$ ( $12 \mathrm{~min}$ in the high intensity range) the load is increased with $10 \%$. If necessary fine-tuning can be done using steps of $5 \%$.

Resistance training is composed as 2 series of 10 repetitions of 6 exercises targeting all major muscle groups).

- The strength training consists of two series of 10 repetitions of six exercises: leg press, chest press, abdominal crunches floor, lat pull down, low row and step up.

$\circ$ In week 1 using $65 \%$ of calculated 1RM (baseline)

○ In week 2 using $70 \%$ of calculated 1RM (baseline)

○ In week 3 using $65 \%$ of calculated 1RM (at 3 weeks).

$\circ$ In week 4 using $70 \%$ of calculated 1 RM (at 3 weeks)

- The 1RM will be determined at baseline and at 3 weeks using. The strength exercises are performed according to: $2 \mathrm{~s}$ of concentric strength and $2 \mathrm{~s}$ of eccentric strength. The 1RM is calculated by the Brzycki formula:

$$
\begin{aligned}
& 1 R M=W^{*} 36 /(37-r) \\
& W=\text { weight in kilograms } \\
& r=\text { repetitions }
\end{aligned}
$$

- The last bout with 10 repetitions needs to be attainable. If this is not the case in the next session dosing will be $5-10 \%$ lower. If in the last bout it appears that exercises are to low ( $\geq 15$ repetitions will be achieved), in the next sessions dosing will be 5-10\% higher.

Patients will also be given instructions about how to conduct aerobic exercises at home. Patients are instructed to aim at 60 min walking and/or cycling a day, but at least $30 \mathrm{~min}$ a day. If possible, patients can do more than $60 \mathrm{~min}$ of walking or cycling every day. In case of a low exercise capacity it is advised to walk/cycle 2-3 times a day for periods of 10-20 min. Also an electric bicycle, a stationary bicycle and/or a walking aid (walker) is allowed if necessary.

Patients in both the intervention and control group will wear an accelerometer for four weeks to count the number of steps walked in order to record the overall activity.

The conventional wisdom is that training blocks of 3-5 min are particularly effective in terms of enhancing exercise capacity. Most of the nine studies that produced the largest increases in $\mathrm{VO}_{2} \max (\sim 0.85 /$ min) used blocks of 3-5 min and HIT. Many of these studies presented either individual data or ranges for $\mathrm{VO}_{2} \mathrm{max}$ values pre- and post-training, and an appraisal of these data suggests that a marked training response was seen in all subjects [29].

In the intervention group we expect to achieve the following improvements after four weeks of prehabilitation compared to baseline measurements: a $10 \%$ increase in $\mathrm{VO} 2$ peak, a $15 \%$ increase in $\mathrm{VO} 2$ at anaerobic level, a $20-40 \%$ increase in 1-RM tests and an increase of $>20$ $\mathrm{m}$ in the 6MWT [12, 15, 16, 19, 27].

\section{Nutritional assessment and intervention}

The nutritionist will complete nutritional assessments at baseline appointments and during the prehabilitation program using the patient-generated subjective global assessment (PG-SGA), body composition (skinfold measurements, mid upper-arm muscle area), hand grip strength and nutritional intake (caloric and protein intake), and a patients' three-day food diary.

We aim to establish an anabolic condition preoperatively. In cachectic and sarcopenic patients we try to increase lean body mass by $1-2 \mathrm{~kg}$ or more during four weeks of prehabilitation. In order to achieve this goal, the target dietary protein intake will be $1.5-1.8 \mathrm{~g} / \mathrm{kg}$ body weight in all patients [16, 30-35].

Participants will receive high-quality protein supplements containing $30 \mathrm{~g}$ of whey protein following exercise and before sleep. Dietary advice will be given in order to achieve adequate oral protein intake spread properly across meals. Since vitamin D is associated with muscle mass and muscle strength [36, 37], vitamin D will be supplemented daily according to guidelines of the Health Council of the Netherlands $(10 \mu \mathrm{g}$ for women aged $50-69 y$, for men $<70 y$ and women $<50 y$ with colored skin and/or little sun exposure and $20 \mu \mathrm{g}$ for women and men aged 70 y or older) [38]. Besides vitamin D, many elderly patients may have other micronutrient deficiencies or ingest vitamins and minerals below recommended doses 
before and after surgery. Therefore, all other vitamins and minerals are supplied in a multivitamin/mineral supplement containing $50 \%$ of the recommended daily allowance.

During the period of hospitalization, the time (in days) that patients consume nil per mouth is recorded. Also, on the day of discharge, a trained dietician performs a 24-h recall questionnaire to estimate oral protein- and energy intake. Nutritional status assessment (PG-SGA) will be performed at 4 and 8 weeks post-surgery by an investigator - trained by a registered dietician.

\section{Smoking cessation}

A smoking cessation program with intensive counseling and nicotine replacement therapy (NRT) will be offered to all patients during the weeks of prehabilitation. Past and current smoking is measured using questionnaires. Counselling includes group weekly group sessions and telephone calls. NRT may consist of any type of replacement therapy. Approximately $15-20 \%$ of our patients are smokers when cancer is diagnosed [13]. The goal is to achieve a smoking cessation rate of $80 \%$ before surgery.

\section{Psychological coping}

It is expected that patients undergoing surgery for cancer are anxious with some component of depression. Since both anxiety and depression can influence the motivation to carry out social and functional activities, psychological strategies can be put in place to help patients to cope with the stress of surgery and disease. Therefore, patients will be screened for anxiety and depression using the GAD-7 and PHQ-9 questionnaires. If these questionnaires result in a high score (GAD-7 of 10 or higher; PHQ- 9 score 15 or higher), patients are considered high-risk and will be offered a referral to a psychologist. Referred patients will receive a total of $1.5 \mathrm{~h}$ of psychological intervention in the first session and more sessions during the 4 weeks of prehabilitation if necessary.

All patients in the intervention group will be given instructions on relaxation and breathing techniques by a trained investigator. They will be given an instruction $\mathrm{CD}$, which they can use for relaxation techniques at home. After the program, patients will be asked if their perceived usefulness of these techniques. For psychological support, the intervention group will be contacted weekly by the investigator by phone. During these 5-15 min phone calls, a researcher will shortly evaluate personal progression by a standardized set of questions. In order to enhance adherence to the prehabilitation program, all patients in the intervention group will receive an instructional brochure that includes information about all elements of the program.

\section{Study outcomes}

The initial primary outcome will be postoperative complications, as scored by the Comprehensive Complication Index [39], with the relevant data being collected as a continuous variable and calculated using the sum of morbidity and mortality presented on the Clavien Dindo classification [40]. The CCI score will be calculated at the 30 days of follow-up. The second primary outcome will be the $6 \mathrm{MWT}$ measured at 4 weeks after surgery and compared to baseline. The 6MWT will additionally be measured directly after prehabilitation and 8 weeks after surgery.

Secondary outcomes will include patient reported outcome measurements (PROMs) such as health related quality of life (HRQoL) (EORTC QLQ-CR29 and EORTC QLQ-C30 and RAND questionnaires) and depression and anxiety scores (GAD-7, PHQ-9 questionnaires), functional capacity (CPET including VO2max, VO2peak, AT, the sit to stand test, stair climb test, hand grip strength and activity questionnaire), nutritional status (3-day food diary, PG-SGA, anthropometry), postoperative complications, length of hospital stay, study compliance, patient satisfaction and a cost-effectiveness analysis. All secondary outcomes are measured at baseline, the week before surgery, 4 and 8 weeks and 1 year post-surgery.

\section{Statistical analysis}

Baseline characteristics of both groups will be compared to assess the adequacy of the randomization. Data will be analyzed on an intention-to-treat basis. In addition, a per-protocol analysis will be performed. Trial results will be published in a respective journal. Primary and secondary outcomes for the intervention and control groups will be compared.

The primary outcome CCI will be described as the mean plus the standard deviation (SD). Since we expect $\mathrm{CCI}$ to be right skewed we will also describe $\mathrm{CCI}$ as the median plus interquartile range (IQR) and percentage above 20. To test the hypothesis (H0) that the study arms result in similar CCIs (in other words, prehabilitation does not prevent postoperative complications), we will use the Student's T-test if data are normally distributed. Mann-Whitney $U$ if data are not normally distributed or statistical methods that take into account the possible zero-inflated nature of the data. The second primary outcome $6 \mathrm{MWT}$ is a continuous variable. This data will be stated as means, plus SD, at each time point. To accommodate the repeat measurements for individuals, we will use a generalized linear mixed model to statistically test the hypothesis of both study arms being equal in terms of functional capacity over time.

All secondary outcomes will be described as means plus SD or median plus IQR, with the data being 
continuous, and measures for each time point being normally and non-normally distributed respectively. Categorical parameters will be described as number plus percentage per time point. Statistical methods will include t-test and the Mann-Whitney $U$ test for continuous parameters, distributed either normally or not normally respectively, at a single postoperative time-point. Categorical outcomes will be analyzed with Chi-square testing or regression analysis (logistic, ordinal or nominal, depending on the definition of the parameter) for single time points. The size of the sample will be calculated on the basis of the primary aim: the reduction of postoperative complications as determined with the CCI score. With our population variables, the CCI mean is 10.4 (SD 14), and the target reduction is $30 \%$. We use an alpha of 0.05 and power of 0.80 (two-sided test). We expect a dropout rate of $10 \%$. We therefore need 714 patients: 357 in each arm. This gives us sufficient statistical power to demonstrate the expected proportion of difference (55\% versus $20 \%$ ) in the 6MWT between baseline and surgery based on previous studies [14, 27]. Approximately 600 eligible CRC patients undergo surgery in one of the six participating hospitals annually. This implies that we will complete inclusion within two years with the inclusion period being followed by a year of follow-up.

Due to limited clinical data regarding effect size of the primary endpoint - CCI - an interim analysis will be performed. The interim analysis is planned if half of the intended number of subjects have completed the 4 week assessment (i.e. timing of the primary endpoint assessment). The intention of the interim analysis is to terminate the study if there is a statistical significant difference between study arms.

\section{Economic evaluation}

To analyze cost-effectiveness, we will focus on the results of the program defined as reduction of complications, improving survival, less need for postoperative care and improvements in social productivity. The economic evaluation will be performed per participating center and includes incremental cost-effectiveness and cost-utility analysis using the RAND and iMTA-PCQ questionnaires. The cost-effectiveness ratio will be calculated by dividing the difference between the mean total costs for the exercise and control groups by the difference in the mean effect in the groups [41]. The cost-utility ratio expresses the additional costs of the intervention compared with the control group per quality-adjusted life years [42].

\section{Discussion}

Despite advances in surgical techniques and improvements in postoperative care, morbidity and mortality remain high in CRC patients undergoing surgery. Postoperative complications occur in up to $50 \%$ of patients and surgery is associated with a 20 to $40 \%$ reduction in physiological and functional capacity [2-5].

Since we know that the number and severity of complications are, or may be, associated with preoperative functional capacity, nutritional status, smoking behavior and psychological well-being, it is incumbent on us to test a multimodal intervention program that targets these issues. Traditional approaches have focused on the postoperative period for rehabilitation and lifestyle changes. However, recent evidence has shown that the preoperative period is a better time to intervene [27]. Therefore, we initiated the first international randomized controlled trial on multimodal prehabilitation for patients undergoing colorectal surgery for cancer.

Patient lifestyle (stated as inactivity, obesity, dietary pattern, and smoking behavior) is an important contributor to the development of CRC [43]. Moreover, CRC patients often develop problems with their nutritional status which may aggravate deconditioning and muscle wasting (sarcopenia) [31, 32, 44]. This implies that, particularly in this group of patients, there is considerable potential for improvement in both nutritional status and functional exercise capacity [45-47]. A recent review indicated that optimizing functional exercise capacity in the surgical population can, by comparison with controls, result in fewer postoperative complications, shorten the length of hospital stay, reduce disability, and improve quality of life [48]. However, there have been no previous studies rigorously evaluating the impact of multimodal prehabilitation prior to digestive surgery.

Interventions that involve physical exercise training for endurance and strength, nutrition, mental support and smoking behavior have all found an independent and clinically relevant effect on the reduction of postoperative complications in small studies. If all these interventions are orchestrated in an innovative prehabilitation program, it may prove feasible to design a highly effective and comprehensive intervention. Synergy may result from these individual interventions if they are applied in a multimodal program since it is known that protein supplements one hour after exercise improve uptake and enhance anabolic effects [30-33, 44, 49]. Nutritional supplementation four weeks before and after surgery has been shown to enhance preoperative functional walking capacity and recovery in patients undergoing colorectal resection for cancer [34]. Moreover, the release of dopamine during exercise improves the psychological mindset and smoking cessation improves the ability to perform exercise.

After the diagnosis of CRC, there is a relatively short period of 4-5 weeks before the actual surgery. A rigorous intervention program of prehabilitation is therefore 
required that is closely coordinated with the entire medical treatment program. An additional potential benefit is the empowerment of patients, who may then play an active role in coping with their disease. From a physiological point of view and based on limited practical experience, it seems feasible to achieve clinical relevant effects during the period of four weeks between diagnosis and surgery. However, this is possible only with a combination of robust innovative interventions involving nutrition, physiological support, smoking cessation and exercise training. These ideas are supported by Carli et al., who stated that a multidisciplinary prehabilitation program needs to be developed, tested, implemented and delivered to patients $[16,35]$.

Another reason why optimal recovery after surgery is important is because it will increase the potential of patients to withstand additional therapies such as chemotherapy, targeted immunotherapy, metastatic disease resection and/or hyperthermic intraperitoneal chemotherapy (HIPEC) [50, 51]. A study by the Dutch cancer register included 11.000 stage-3 CRC patients (20082013) [7]. 4899 of whom were not treated with chemotherapy. The five-year survival rate in this group was only $39 \%$. If chemotherapy started $>12$ weeks postoperatively, the five-year survival rate increased to $54 \%$. When chemotherapy began $<6$ weeks after the operation, this rate increased further to $76 \%$. Improved functional capacity may facilitate an earlier start of adjuvant chemotherapy and thereby increasing survival an improving HRQoL $[7,8,52,53]$.

Despite previous evidence from small-scale trials, there are currently no standardized prehabilitation programs and they are therefore not mentioned in current medical guidelines. This highlights the need to design, test, optimize, and implement a multimodal program for maximizing improvements in nutritional status and functional capacity prior to surgery. We expect to see a reduction in postoperative complications, Length Of hospital Stay (LOS), intensive care stay, 30-day mortality rate, and health expenditure due to the multimodal prehabilitation program. The sum of all these separate outcomes will be measured using the Comprehensive Complication Index (CCI), which is a relatively new and interesting outcome measure [40]. As stated in the literature, patients with postoperative complications report a lower HRQoL than patients without. We will be the first to determine whether the HRQoL of patients with postoperative complications is inferior both preoperatively and 1 year postoperatively than the HRQoL of patients without complications. We expect poorer HRQoL in patients with complications preoperatively and one year postoperatively than in patients without complications.

Limitations of the study should be noted as well. Due to its nature this study is performed non-blinded. This may result in higher dropout rates or an increased activity level in the control group, due to the growing and intuitive understanding of the benefits of exercise training and optimal nutrition. To limit this potential bias of increased activity, we will introduce activity trackers to all patients. We do realize patient characteristics, social circumstances and healthcare facilities will not be the same in all countries. Therefore, we will stratify per participating center. Our international approach of the randomized controlled trial will demonstrate that a worldwide implementation may be possible. In case our multimodal program proves to enhance postoperative outcome, it will be impossible to discover which element of the program attributed most. A large sample size and different outcome measurement will facilitate subgroup analyses, to determine effects of the program within different areas, such as functional capacity, body composition, and quality of life. Also, to allow for comparison of the effects of single modal interventions in this multimodal program. Our large sample size will also give us the possibility to analyze the possible effect differences of an intensive hospital-based prehabilitation program in different patient groups. This way, prehabilitation in the current form can be implemented for patient groups who will benefit the most from intensive hospital-based training. For less high-risk patients, future research could focus on programs in which training is less intensively monitored, such as home-based programs.

Our study investigating prehabilitation is a good example of research in prevention and it will be the first to systematically implement existing knowledge from a variety of different medical specialties and basic science into a four-element multimodal preoperative program for CRC patients with the aim of improving functional capacity and reducing the postoperative complication rate.

\footnotetext{
Abbreviations

6-MWT: Six minute walk test; CAL: Colorectal anastomotic leakage;

CCl: Comprehensive complication index; CHAMPS: Community health activities model program for seniors; CPET: CardioPulmonary exercise testing; CRC: ColoRectal cancer; DSMB: Data safety monitoring board; ERAS: Enhanced recovery after surgery; GAD-7: Generalized anxiety disorder 7; HIPEC: Hyperthermic intraPEritoneal chemotherapy; HIT: High intensity training; HRQoL: Health related quality of life; LOS: Length of hospital stay; MMC: Máxima medical center; NRT: Nicotine replacement therapy; PG-SGA: Patient generated subjective global assessment; PHQ-9: Patient health questionnaire 9; SD: Standard deviation
}

\section{Acknowledgements}

The authors thank Prof. L van Loon of the department of Human Movement Sciences Maastricht University Medical Center, and Prof. L van de Pol and Dr. O. Husson of the Netherlands Comprehensive Cancer Organisation, for their support in writing this study protocol. This is an investigator initiated study. Registered trial: NTR5947. Medical ethical reviewing committee Máxima Medical Center number: NL58281.015.16. 


\section{Funding}

Each participating center is responsible for their own funding and funded from their own budget. Máxima Medical Center is additionally supported by the Dutch Cancer Society (KWF), for local organization costs and materials used in the study. The KWF has peer reviewed the protocol to grant the subsidy, however they did not have impact on the design of the study. The Peri-Operative Program (POP) will provide funding for the research conducted by the McGill group in the Montréal General Hospital. The funding sources had no role in the design of this study and will not have any role during its execution, analyses, interpretation of the data, or decision to submit results.

\section{Availability of data and materials}

Not applicable.

\section{Trial status}

Open for inclusion since June 2017.

\section{Authors' contributions}

SR and FC conceived the study. SR, FC, SD, SB, RL, RR, GS, GDS drafted the manuscript. SR, FC, SD, RL, RA, EM, CS, GS, GDS designed the study. SR, FC, $S D, G T, R B, M G, N B, R A, E M, S B, G M, R L, C F, I G, J C, C S, R R, G S, G D S$, are local investigators at the participating centers. The study is supervised and coordinated by SR, FC, SD and GDS. GDS is responsible for accurate execution of the study. All authors approved the final manuscript.

\section{Authors' information}

$\mathrm{SJ}$ is a PhD candidate in surgery and surgical oncology at Máxima Medical Center, Veldhoven, the Netherlands and at the NUTRIM School of Nutrition and Translational Research in Metabolism, Maastricht, The Netherlands. FC is a Professor in anesthesiology at the Montréal General Hospital and McGill University, Montréal, Canada.

SD is a senior researcher at the Danish Cancer Society Research Center, Copenhagen, Denmark.

GT is a PhD candidate in surgery and surgical oncology at Máxima Medical Center, Veldhoven, the Netherlands.

$\mathrm{RB}$ is a PhD candidate and surgical resident at the Center for Surgical Science, Zealand University Hospital, Køge and Slagelse, Denmark.

MG is an anesthesiologist at Foch Hôpital, Paris, France.

NB is a sports physician at Foch Hôpital, Paris, France.

RA is a research coordinator and kinesiologist at the Montréal General Hospital, Montréal, Canada.

EM is a PhD candidate at the department of anesthesiology at the Montréal General Hospital, Montréal, Canada.

$\mathrm{SB}$ is a senior researcher at the Netherlands Comprehensive Cancer Organisation, Utrecht, the Netherlands.

GM is an anesthesiologist at the department of anesthesiology at the Hospital Clinic de Barcelona, IDIBAPS, University of Barcelona, Spain. RL is a dietician at the department of nutrition at Máxima Medical Center, Veldhoven, the Netherlands.

CF is a Professor in surgery at the Ferrara University Hospital, Ferrara, Italy. IG is a Professor in surgery at Center for Surgical Science, Zealand University Hospital, Køge and Roskilde, Denmark.

$\mathrm{CJ}$ is a Professor in research at the department of oncology at the Finsen Centre, Rigshospitalet and the Danish Cancer Society Research Center, Copenhagen, Denmark.

CS is a kinesiologist at the Montréal General Hospital and the McGill University, Montréal, Canada.

RR is a surgeon at Máxima Medical Center, Veldhoven, the Netherlands. GS is a sports physician at Máxima Medical Center, Veldhoven, the Netherlands.

GDS is a surgeon at Máxima Medical Center, Veldhoven, the Netherlands.

\section{Ethics approval and consent to participate}

Medical ethical reviewing committee Máxima Medical Center number: NL58281.015.16. Ethical reviewing committees of all participating centers have additionally reviewed and approved the protocol. A written informed consent form for publication will be available for every study participant.

\section{Consent for publication}

Written informed consent has been obtained from all study participants.

\section{Competing interests}

The authors declare that they have no competing interests.

\section{Publisher's Note}

Springer Nature remains neutral with regard to jurisdictional claims in published maps and institutional affiliations.

\section{Author details}

'Department of Surgical Oncology, Máxima Medical Center, Veldhoven, the Netherlands. ${ }^{2}$ Department of Anesthesiology, the Montréal General Hospital, McGill University, Montréal, Canada. ${ }^{3}$ Danish Cancer Society Research Center, Copenhagen, Denmark. ${ }^{4}$ Department of Surgery, Center for Surgical Science, Zealand University Hospital, Køge, Denmark. ${ }^{5}$ Department of Anesthesiology, Foch Hôpital, Paris, France. ${ }^{6}$ Department of Sports Medicine, Foch Hôpital, Paris, France. ${ }^{7}$ Netherlands Comprehensive Cancer Organisation, Utrecht, the Netherlands. ${ }^{8}$ Department of Anesthesiology, Hospital Clinic de Barcelona, IDIBAPS, University of Barcelona, Barcelona, Spain. ${ }^{9}$ Department of Nutrition, Máxima Medical Center, Veldhoven, the Netherlands. ${ }^{10}$ Department of Surgery, S. Anna University Hospital, Ferrara, Italy. ${ }^{11}$ Department of Oncology, Finsen Center, Rigshospitalet, Copenhagen, Denmark. ${ }^{12}$ Department of Kinesiology and Physical Education, McGill University, Montréal, Canada. ${ }^{13}$ Department of Sports Medicine, Máxima Medical Center, Veldhoven, the Netherlands. ${ }^{14}$ Department of Surgery, Máxima Medical Center, P.O. Box 7777, Veldhoven, the Netherlands.

Received: 2 February 2017 Accepted: 19 December 2018

Published online: 22 January 2019

\section{References}

1. Torre LA, Bray F, Siegel RL, Ferlay J, Lortet-Tieulent J, Jemal A. Global Cancer statistics, 2012. CA Cancer J Clin. 2015;65.

2. Kirchhoff $P$, Clavien PA, Hahnloser D. Complications in colorectal surgery risk factors and preventive strategies. Patient Saf Surg 2010, 45.

3. McDermott FD, Heeney A, Kelly ME, Steele RJ, Carlson GL, Winter DC. Systematic review of preoperative, intraoperative and postoperative risk factors for colorectal anastomotic leaks. Br J Surg. 2015 Apr;102(5).

4. Govaert JA, Fiocco M, van Dijk WA, Scheffer AC, de Graaf EJ, Tollenaar RA, Wouters MW. Costs of complications after colorectal cancer surgery in the Netherlands. Building the business case for hospitals. Eur J Surg Oncol. 2015;41(8).

5. Lawrence VA, Hazuda HP, Cornell JE, Pederson T, Bradshaw PT, Mulrow CD, et al. Functional independence after major abdominal surgery in the elderly. J Am Coll Surg. 2004;199:762-72.

6. Christensen T, Kehlet H. Postoperative fatigue. World J Surg. 1993;17(2).

7. Bos AC, van Erning FN, van Gestel YR, Creemers GJ, Punt CJ, van Oijen MG, Lemmens VE. Timing of adjuvant chemotherapy and its relation to survival among patients with stage III colon cancer. Eur J Cancer. 2015 Nov;51(17).

8. Sun Z, Adam MA, Kim J, Nussbaum DP, Benrashid E, Mantyh CR, Migaly J. Determining the optimal timing for initiation of adjuvant chemotherapy after resection for stage II and III Colon Cancer. Dis Colon Rectum. 2016; 59(2):87-93.

9. Kehlet H. Manipulation of the metabolic response in clinical practice. World J Surg. 2000;24.

10. Van Rooijen SJ, Huisman D, Stuijvenberg M, Stens J, Roumen RMH, Daams F, Slooter GD. Intraoperative modifiable risk factors of colorectal anastomotic leakage: why surgeons and anesthesiologists should act together. Int J Surg. 2016;36.

11. Kehlet H, Wilmore DW. Evidence-based surgical care and the evolution of fast-track surgery. Ann Surg. 2008;248(2).

12. Debes C, Aissou M, Prehabilitation BM. Preparing patients for surgery to improve functional recovery and reduce postoperative morbidity. Ann $\mathrm{Fr}$ Anesth Reanim. 2014;33(1)

13. Van Rooijen SJ, Carli F, Oksbjerg Dalton S, Johansen C, Dieleman J, Roumen $\mathrm{RMH}$, Slooter GD. Preoperative modifiable risk factors in colorectal surgery: an observational cohort study identifying the possible value of prehabilitation. Acta Oncol. 2017.

14. Steffens D, Beckenkamp PR, Hancock M, Solomon M, Young J. Preoperative exercise halves the postoperative complication rate in patients with lung cancer: a systematic review of the effect of exercise on complications, length of stay and quality of life in patients with cancer. Br J Sports Med. 2018;52(5):344. 
15. Li C, Carli F, Lee L, Charlebois P, Stein B, Liberman AS, Kaneva P, Augustin B, Wongvingsinn M, Gamsa A, Kim DJ, Vassiliou MC, Feldman LS. Impact of a trimodal prehabilitation program on functional recovery after colorectal cancer surgery: a pilot study. Surg Endosc. 2013;27(4).

16. Carli F, Charlebois P, Stein B, Feldman L, Zavorsky G, Kim DJ, Scott S, Mayo NE. Randomized clinical trial of prehabilitation in colorectal surgery. BJS. 2010;97.

17. Harkness K, Morrow L, Smith K, Kiczula M, Arthur HM. The effect of early education on patient anxiety while waiting for elective cardiac catheterization. Eur J Cardiovasc Nurs. 2003;2(2).

18. Snowden CP, Prentis JM, Anderson HL, Roberts DR, Randles D, Renton M, Manas DM. Submaximal cardiopulmonary exercise testing predicts complications and hospital length of stay in patients undergoing major elective surgery. Ann Surg. 2010;251(3).

19. Barberan-Garcia A, Ubré M, Roca J, Lacy AM, Burgos F, Risco R, Momblán D, Balust J, Blanco I, Martínez-Pallí G. Personalised Prehabilitation in high-risk patients undergoing elective major abdominal surgery: a randomized blinded controlled trial. Ann Surg. 2018;267(1):50-6.

20. Mayo NE, Feldman L, Scott S, Zavorsky G, Kim DJ, Charlebois P, Stein B, Carli F. Impact of preoperative change in physical function on postoperative recovery, argument supporting prehabilitation for colorectal surgery. Surgery. 2011;150(3)

21. Munafo MR. Perioperative anxiety and postoperative pain. Psychol Health Med. 1998:3.

22. Rosenberger PH, Jokl P, Ickovics J. Psychosocial factors and surgical outcomes: an evidence-based literature review. J Am Acad Orthop Surg. 2006;14

23. Wallace LM. Psychological preparation as a method of reducing the stress of surgery. J Hum Stress. 1984;10.

24. Munafo MR, Stevenson J. Anxiety and surgical recovery: reinterpreting the literature. J Psychosom Res. 2001;51

25. Kiecolt-Glazer JK, Page GG, Marucha PT, MacCallum RC, Glaser R. Psychological influences on surgical recovery: perspectives from psychoneurommunology. Am Psychol. 1998;53.

26. Gillis C, Li C, Lee L, Awasthi R, Augustin B, Gamsa A, Liberman AS, Stein B, Charlebois P, Feldman LS, Carli F. Prehabilitation versus rehabilitation: a randomized control trial in patients undergoing colorectal resection for cancer. Anesthesiology. 2014;121(5).

27. Fearon $\mathrm{KC}$, Ljungqvist $\mathrm{O}$, Von Meyenfeldt $\mathrm{M}$, Revhaug $\mathrm{A}$, Dejong $\mathrm{CH}$, Lassen K, Nygren J, Hausel J, Soop M, Andersen J, Kehlet H. Enhanced recovery after surgery: a consensus review of clinical care for patients undergoing colonic resection. Clin Nutr. 2005;24.

28. Clinical exercise testing with reference to lung diseases: indications, standardization and interpretation strategies. ERS Task Force on Standardization of Clinical Exercise Testing. European Respiratory Society. Eur Respir J 1997, 10:2662-2689.

29. Bacon AP, Carter RE, Ogle EA, Joyner MJ. $\mathrm{VO}_{2}$ max trainability and high intensity interval training in humans: a meta-analysis. PLoS One. 2013;8(9).

30. van Loon L. Role of dietary protein in post exercise muscle reconditioning Nestle Nutr Inst Workshop Ser. 2013;75.

31. Paddon-Jones D, Campbell WW, Jacques PF, Kritchevsky SB, Moore LL, Rodriguez NR, Van Loon L. Protein and healthy aging. Am J Clin Nutr. 2015; 101(6).

32. Cermak NM, Res PT, De Groot LC, Saris WH, Van Loon LJ. Protein supplementation augments the adaptive response of skeletal muscle to resistance-type exercise training: a meta-analysis. Am J Clin Nutr. 2012;96(6).

33. Wall BT, Snijders T, Senden JM, Ottenbros CL, Gijsen AP, Verdijk LB, van Loon LJ. Disuse impairs the muscle protein synthetic response to protein ingestion in healthy men. J Clin Endocrinol Metab. 2013:98(12).

34. Gillis C, Loiselle SE, Fiore JF Jr, Awasthi R, Wykes L, Liberman AS, Stein B. Prehabilitation with whey protein supplementation on perioperative functional exercise capacity in patients undergoing colorectal resection for Cancer: a pilot double-blinded randomized placebo-controlled trial. J Acad Nutr Diet. 2016;116(5)

35. Carli F, Scheede-Bergdahl C. Prehabilitation to enhance perioperative care. Anesthesiol Clin. 2015;33(1).

36. Salles J, Chanet A, Giraudet C, Patrac V, Pierre P, Jourdan M, Luiking YC, Verlaan S, Migne' C, Boirie $Y$, et al. 1,25(OH)(2)-vitamin D(3) enhances the stimulating effect of leucine and insulin on protein synthesis rate through Akt/PKB and mTOR mediated pathways in murine C2C12 skeletal myotubes. Mol Nutr Food Res. 2013;57:2137-46.

37. Mithal A, Bonjour JP, Boonen S, Burckhardt P, Degens H, El Haij Fuleihan G, Josse R, Lips P, Morales Torres J, Rizzoli R, et al. IOF CSA nutrition working group. Impact of nutrition on muscle mass, strength, and performance in older adults. Osteoporos Int. 2013;24:1555-66.

38. Voedingsnormen vitamine D, 2015 (www.voedingscentrum.nl).

39. Slankamenac K, Nederlof N, Pessaux P, de Jonge J, Clavien P. The CCl, a novel and more sensitive endpoint for assessing outcome and reducing sample size in RCTs. Ann Surg. 2014;260(5).

40. Dindo D, Demartines N, Clavien PA. Classification of surgical complications: a new proposal with evaluation in a cohort of 6336 patients and results of a survey. Ann Surg. 2004;240(2).

41. Drummond MF, Sculpher MJ, Torrance GW, O'Brien BJ, Stoddart GL. Methods for the economic evaluation of health care programmes. New York: Oxford University Press; 2005

42. Stinnett AA, Mullahy J. Net health benefits: a new framework for the analysis of uncertainty in cost-effectiveness analysis. Med Decis Mak. 1998; 18(2 Suppl).

43. Cappellani A, Zanghi A, Di Vita M, Cavallarao A, Piccolo G, Veroux P, Lo Menzo E, Cavallaro V, De Paoli P, Veroux M, Berretta M. Strong correlation between diet and development of colorectal cancer. Front Biosci. 2013;18(1).

44. Pennings B, Koopman R, Beelen M, Senden JM, Saris WH, van Loon JC. Exercising before protein intake allows for greater use of dietary proteinderived amino acids for de novo muscle protein synthesis in both young and elderly men. Am J Clin Nutr Feb. 2011:93(2).

45. Fong DY, Ho JW, Hui BP, Lee AM, Macfarlane DJ, Leung SS, Cerin E, Chan WY, Leung IP, Lam SH, Taylor AJ, Cheng KK. Physical activity for cancer survivors: meta-analysis of randomised controlled trials. BMJ. 2012;344.

46. Mishra SI, Scherer RW, Snyder C, Geigle P, Gotay C. Are exercise programs effective for improving health-related quality of life among cancer survivors? A systematic review and meta-analysis. Oncol Nurs Forum. 2014;41(6).

47. Mishra SI, Scherer RW, Snyder C, Geigle PM, Berlanstein DR, Topaloglu O. Exercise interventions on health-related quality of life for people with cancer during active treatment. Clinical otolaryngology: official journal of ENT-UK; official journal of Netherlands Society for Oto-Rhino-Laryngology \& Cervico-facial Surgery, 2012; 37(5).

48. Carli F, Zavorsky G. Optimizing functional exercise capacity in the elderly surgical population. Curr. Opin. Clin. Nutr. Metab. Care. 2005;8(1).

49. Tieland $M$, van de Rest $O$, Dirks ML, van der Zwaluw N, Mensink M, van Loon LJ, de Groot LC. Protein supplementation improves physical performance in frail elderly people: a randomized, double-blind, placebocontrolled trial. J Am Med Dir Assoc Oct. 2012;13(8).

50. van Waart H, Stuiver MM, van Harten WH, Sonke GS, Aaronson NK. Design of the Physical exercise during adjuvant chemotherapy effectiveness study (PACES): a randomized controlled trial to evaluate effectiveness and costeffectiveness of physical exercise in improving physical fitness and reducing fatigue. BMC Cancer. 2010;10.

51. van Rooijen SJ, Engelen MA, Scheede-Bergdahl C, et al. Systematic review of exercise training in colorectal cancer patients during treatment. Scand J Med Sci Sports. 2017;00:1-11.

52. De Backer IC, Van Breda E, Vreugdenhil A, Nijziel MR, Kester AD, Schep G. High-intensity strength training improves quality of life in cancer survivors. Acta Oncol. 2007:46(8).

53. Mols O, Beijers AJ, Vreugdenhil G, Verhulst A, Schep G, Husson O. Chemotherapy-induced peripheral neuropathy, physical activity and healthrelated quality of life among colorectal cancer survivors from the PROFILES registry. J Cancer Surviv. 2015;9(3).

\section{Ready to submit your research? Choose BMC and benefit from:}

- fast, convenient online submission

- thorough peer review by experienced researchers in your field

- rapid publication on acceptance

- support for research data, including large and complex data types

- gold Open Access which fosters wider collaboration and increased citations

- maximum visibility for your research: over $100 \mathrm{M}$ website views per year

At $\mathrm{BMC}$, research is always in progress.

Learn more biomedcentral.com/submission 